\title{
Search for a TeV gamma-ray halo of Mkn 501
}

\author{
F. A. Aharonian ${ }^{1}$, A. G. Akhperjanian ${ }^{7}$, J. A. Barrio ${ }^{2,3}$, K. Bernlöhr ${ }^{1}$, O. Bolz ${ }^{1}$, H. Börst ${ }^{5}$, H. Bojahr ${ }^{6}$,
} J. L. Contreras ${ }^{3}$, J. Cortina ${ }^{2}$, S. Denninghoff ${ }^{2}$, V. Fonseca ${ }^{3}$, J. C. Gonzalez ${ }^{3}$, N. Götting ${ }^{4}$, G. Heinzelmann ${ }^{4}$, G. Hermann ${ }^{1}$, A. Heusler ${ }^{1}$, W. Hofmann ${ }^{1}$, D. Horns ${ }^{4}$, A. Ibarra ${ }^{3}$, C. Iserlohe ${ }^{6}$, I. Jung ${ }^{1}$, R. Kankanyan ${ }^{1,7}$, M. Kestel ${ }^{2}$, J. Kettler ${ }^{1}$, A. Kohnle ${ }^{1}$, A. Konopelko ${ }^{1, \S}$, H. Kornmeyer ${ }^{2}$, D. Kranich ${ }^{2}$, H. Krawczynski ${ }^{1}$, H. Lampeitl ${ }^{1}$, E. Lorenz ${ }^{2}$, F. Lucarelli ${ }^{3}$, N. Magnussen ${ }^{6}$, O. Mang ${ }^{5}$, H. Meyer ${ }^{6}$, R. Mirzoyan ${ }^{2}$, A. Moralejo ${ }^{3}$, L. Padilla ${ }^{3}$, M. Panter ${ }^{1}$, R. Plaga ${ }^{2}$, A. Plyasheshnikov, ${ }^{1,}$, J. Prahl $^{4}$, G. Pühlhofer ${ }^{1}$, W. Rhode ${ }^{6}$, A. Röhring ${ }^{4}$, G. P. Rowell ${ }^{1}$, V. Sahakian ${ }^{7}$, M. Samorski ${ }^{5}$, M. Schilling ${ }^{5}$, F. Schröder ${ }^{6}$, M. Siems ${ }^{5}$, W. Stamm ${ }^{5}$, M. Tluczykont ${ }^{4}$, H. J. Völk ${ }^{1}$, C. Wiedner ${ }^{1}$, and W. Wittek ${ }^{2}$

1 Max Planck Institut für Kernphysik, Postfach 103980, 69029 Heidelberg, Germany

2 Max Planck Institut für Physik, Föhringer Ring 6, 80805 München, Germany

3 Universidad Complutense, Facultad de Ciencias Físicas, Ciudad Universitaria, 28040 Madrid, Spain

${ }^{4}$ Universität Hamburg, II. Institut für Experimentalphysik, Luruper Chaussee 149, 22761 Hamburg, Germany

5 Universität Kiel, Institut für Experimentelle und Angewandte Physik, Leibnizstraße 15-19, 24118 Kiel, Germany

6 Universität Wuppertal, Fachbereich Physik, Gaußstr. 20, 42097 Wuppertal, Germany

7 Yerevan Physics Institute, Alikhanian Br. 2, 375036 Yerevan, Armenia

$\S$ On leave from Altai State University, Dimitrov Street 66, 656099 Barnaul, Russia

Received 15 August 2000 / Accepted 10 November 2000

\begin{abstract}
For distant extragalactic sources of gamma-rays in the $\mathrm{PeV}\left(10^{15} \mathrm{eV}\right)$ energy range, interactions of the gamma rays with intergalactic diffuse radiation fields will initiate a pair cascade. Depending on the magnetic fields in the vicinity of the source, the cascade can either result in an isotropic halo around an initially beamed source, or remain more or less collimated. Data recorded by the HEGRA system of imaging atmospheric Cherenkov telescopes are used to derive limits on the halo flux from the AGN Mrk 501. This is achieved by comparing the angular distribution of $\mathrm{TeV}$ gamma-rays during the 1997 burst phase - where direct photons should dominate with the distribution during the 1998/99 quiescent state, where a steady-state halo contribution should be most pronounced. The results depend on the assumptions concerning the angular distribution of the halo; limits on the halo flux within $0.5^{\circ}$ to $1^{\circ}$ from the source range between $0.1 \%$ and $1 \%$ of the peak burst flux.
\end{abstract}

Key words. galaxies: BL Lacertae objects: individual: Mkn 501 - gamma rays: observations

\section{Introduction}

High-energy gamma rays have a limited mean free path in the universe, due to interactions with the intergalactic soft photon radiation, resulting in pair production (Nikishov 1962; Goldreich \& Morrison 1964; Gould \& Schreder 1966; Jelley 1966; Stecker et al. 1992). For (headon) collisions of a gamma ray of energy $E$ with a photon of wavelength $\lambda$, the pair production threshold is crossed if $\lambda<2.4 E(\mu \mathrm{m} / \mathrm{TeV})$. At $\mathrm{PeV}$ energies, interactions with the microwave background radiation limit the mean free path of gamma rays to galactic distance scales. At lower $(\mathrm{TeV})$ energies, infrared or optical photons are required to cross the pair production threshold, and the mean

Send offprint requests to: W. Hofmann, e-mail: Werner.Hofmann@mpi-hd.mpg.de free path rapidly grows with decreasing energy to Mpc scales and beyond. When a source injects very high-energy gamma-rays into this photon background, a cascading process will start, where the gamma ray converts into a pair, the electron and positron in turn Compton-scatter background photons to high energy, which again produce a pair, and so on until the energies are so low that the medium is transparent to these gamma-rays, on the scale of the distance between source and observer (Aharonian \& Atoyan 1985; Protheroe 1986; Zdziarski 1988; Protheroe \& Stanev 1993; Blandford \& Levinson 1995). The cascading process results in universal photon energy spectra, almost independent of the energy of the primary photons. The cascade photons modify the spectrum of direct photons from the source, since cascade photons pile up around the energy where the medium becomes transparent. 
In essence, the energy content in very high-energy photons is transposed into photons of lower energy. To which extent the spectrum of direct photons is modified by the cascade photons depends on the spectral index and on the high-energy cutoff of the primary radiation. If the source emits well into the $\mathrm{PeV}$ region, and if the spectrum resembles a power law $\mathrm{d} N / \mathrm{d} E \sim E^{-\alpha}$ with index $\alpha \approx 2$ - with equal amount of power per decade of energy - the yield of cascade photons is comparable to the number of direct photons. If, on the other hand, the source cuts off at lower energies, or if the primary spectrum is steeper, the energy content of the high-energy photons is small compared to the energy density at lower energies, and the cascade photons represent only a small correction to the spectrum. Already an index of 2.2 suffices to reduce the influence of cascade photons dramatically (Protheroe \& Stanev 1993).

While the discussion given so far concerns the spectral distribution of all photons produced by the source and the cascading effect, relevant e.g. for the description of the diffuse gamma-ray background resulting from the superposition of many unresolved point sources, cascading is also relevant for the angular distribution of photons from individual point sources and may give rise to a halo surrounding distant high-energy sources such as AGNs (Aharonian et al. 1994, 1995). If the magnetic fields in the vicinity of the source are such that the average mean free path between cascade steps - at least during the initial stages of the cascade - is large compared to Larmor radii, the cascade will generate, even for highly beamed primary sources, an isotropic halo. Concerning the intergalactic part of the pair cascade, the required fields are in the range of some nG to some ten $\mathrm{nG}$ (Aharonian et al. 1994), and while little is known about intergalactic magnetic fields, such values are not implausible, and are seen at least in the halos of galaxies and in clusters of galaxies (Kronberg 1996; Eilek 1999). For sources such as the AGNs Mkn 501 or 421 , with characteristic distances around $140 \mathrm{Mpc}$ (for $H=65 \mathrm{~km} \mathrm{~s}^{-1} / \mathrm{Mpc}$ ), halo sizes have been estimated to a few degrees (Aharonian et al. 1994, 1995).

Since the isotropic cascade proceeds over large, typically Mpc scales, the halo intensity will be constant in time, compared to the variable nature of the direct radiation. From simple geometrical arguments, one finds that the characteristic time scale $T$ governing the evolution of the halo flux seen at an angular distance $\theta$ from a source at a distance $d$ is given by

$T \approx \frac{\theta d}{c} \approx 410^{5} \mathrm{y}$

in case the halo is generated in the vicinity of the source; for the numerical estimate $\theta=0.05^{\circ}$ is used, the smallest scale where a halo might be detectable, given the angular resolution of the instrument, and $d=140 \mathrm{Mpc}$. The shortest time scale is possible if the cascades are generated half-way between the source and the observer, in a region of small magnetic fields and low deflection angles,

$T \approx \frac{\theta^{2} d}{2 c} \approx 150 \mathrm{y}$.
One might, therefore, speculate that the very low quiescent-phase TeV gamma-ray flux from AGNs such as Mkn 421 or 501 represents the halo flux, onto which direct photons from burst events are superimposed. In such a scheme, the ratio of the isotropic halo flux $F_{\text {Halo }}$ to the typical flux in bursts $F_{\text {Burst }}$ can be estimated as

$F_{\text {Halo }} / F_{\text {Burst }} \approx r D f$

where $r$ is the ratio of the energy flux in the $\mathrm{PeV}$ and $\mathrm{TeV}$ energy ranges provided by the source, $D$ is the duty cycle, i.e. the fraction of the time the source is in the "burst" state, and $f$ is the beaming fraction, assuming that the observer is located in the center of the beam for burst events. Assuming a flat spectral energy distribution of the source $(r \approx 1)$, a beaming fraction $O(1 / 100)$ and a duty cycle of $O(1 / 10)$ - one predicts a halo flux which is at least a factor of 1000 below the peak flux. On the other hand, if recent data on the strength of the infrared background and their implication concerning the source spectra are taken literally - see e.g. Protheroe \& Meyer (2000) - reconstructed source spectral energy distributions rise steeply at high energy, implying very large values of $r, O\left(10^{2}\right)$ to $O\left(10^{4}\right)$ and a halo flux comparable to the burst flux. Also, smaller intergalactic magnetic fields could result in a beamed halo and a corresponding increase in halo flux. In the extreme case, with fields as low as $10^{-20} \mathrm{G}$, as discussed by Plaga (1995) and Kronberg (1995), the intergalatic part of the cascade is so well collimated that for all practical purposes it cannot be resolved.

This paper describes a search for a $\mathrm{TeV}$ gamma-ray halo surrounding Mkn 501, using the large data base accumulated with the HEGRA system of imaging atmospheric Cherenkov telescopes in the years 1997 through 1999. While Mkn 501 is not the ideal candidate for a halo source - given its relatively modest luminosity and the fact that the "ideal" distance to observe halos is in the range of several $100 \mathrm{Mpc}$ to $1 \mathrm{Gpc}$ (Aharonian et al. 1994), the uncertainties in the model expectation are significant and motivate an experimental search for a gamma-ray halo.

\section{The Mkn 501 data set and the event selection}

The AGN Mkn 501 has been observed on a regular basis during the observation (visibility) periods since 1997 using the HEGRA system of five imaging atmospheric Cherenkov telescopes, located on the Canarian Island of La Palma on the site of the Observatorio del Roque de los Muchachos. The HEGRA telescope system allows the stereoscopic reconstruction of air showers above a threshold of $500 \mathrm{GeV}$, and provides event-by-event a determination of the photon direction with a precision of typically $0.1^{\circ}$, and of the photon energy with a resolution of better than $20 \%$ (Konopelko et al. 1999).

The following analysis is based on the 1997 data set, acquired during the burst phase of Mkn 501 and described in detail by Aharonian et al. (1999a, 1999b), and on the 1998/1999 quiescent-phase data set, described by Aharonian et al. (2000a). With exceptions detailed below, 
the same methods for data selection and event reconstruction were applied to the data sets in this analysis.

In order to search for halo components, it is important to control the point spread function of the instrument and to reduce as much as possible tails in the point spread function. Therefore, the techniques described by Hofmann et al. (2000) were applied to reconstruct the shower geometry from the observed Cherenkov images, providing, for each shower, an error estimate for the direction of the photon. Well-reconstructed photons were then selected by requiring an error of less than $0.1^{\circ}$ in each projection, keeping slightly more than $50 \%$ of all photons. The same approach, with an even tighter selection, has already been applied in a study of the size of the $\mathrm{TeV}$ gamma ray source in the Crab Nebula (Aharonian et al. 2000b).

Past HEGRA studies of the $\mathrm{TeV}$ gamma radiation from Mkn 421 and Mkn 501 (Aharonian et al. 1999a-2000a) or the Crab nebula (Aharonian et al. 1999d) used rather loose cuts to select gamma ray events, in order to minimize systematic errors. For the present study, to gain maximal sensitivity for a faint halo, tighter cuts were applied; the basic idea is to determine the ratio of halo intensity to direct flux with tight cuts, and then derive an absolute halo flux by multiplying this ratio with the absolute flux determined with loose cuts for the direct radiation. Gamma-ray selection cuts are based on the mean scaled width of images (Aharonian et al. 1999a), where gamma-rays appear as a roughly Gaussian peak at 1 with a width of about $10 \%$, whereas nucleonic cosmic rays generate a broad distribution at larger mean scaled width values. Both simulations and Crab and Mkn 501 data show that the best sensitivity for faint sources is achieved with a cut requiring a mean scaled width below 1.05 to 1.10 , compared to cuts at 1.2 to 1.3 in analyses aiming at a precision determination of the flux and spectrum of strong sources. Here, a cut at 1.1 is used, significantly increasing the rejection of cosmic-ray induced events. The slightly increased systematic errors in the determination of the absolute flux ${ }^{1}$ are still negligible in the determination of flux limits, and in any case cancel to a large extent when the ratio of the halo flux to the central direct flux is taken.

\section{Search for a gamma-ray halo}

Depending on the angular distribution of the halo radiation, a halo can manifest itself in different ways in observations with Cherenkov telescopes:

- A component of the halo whose angular spread is very small compared to angular resolution of the instrument - about $4^{\prime}$ for the data set discussed here - will be superimposed onto the direct radiation and cannot be resolved. This limits in particular the detection of the collimated halos generated by cascades in regions of very small magnetic fields;

\footnotetext{
1 For the selection of events used in this analysis, the cut at 1.1 retains $(84 \pm 5) \%$ of all gamma rays, compared to $(97 \pm 2) \%$ for a cut at 1.2 , and $99 \%$ at 1.3 .
}

- A component with an angular spread comparable to the instrumental resolution will result in a widening of the angular distribution, without explicitly resolving the halo;

- A halo with an angular distribution which is wide compared to the resolution can be detected directly; since much of the directional signature is lost in this case, the evaluation of the background due to cosmic rays becomes then a critical issue.

The background mentioned in the third scenario results from the small fraction of charged cosmic rays with a shower evolution similar to that of gamma-ray showers. Since it is uncertain if this remaining background can be modeled with sufficient precision by the simulations, frequently an off-source region is used to measure the background. In case of the HEGRA telescopes, the source is placed $0.5^{\circ}$ off axis in declination, and a region displaced by $0.5^{\circ}$ in the opposite direction is used as a background region. The sign of the displacement alternates from run to run, to minimize systematic effects. Compared to dedicated off-source runs, this method has the advantage that the off-source data are taken at the same time and under exactly the same weather conditions and operating conditions as the on-source data; in addition, the onsource observation time is maximized. A disadvantage of the technique lies in the small separation of $1^{\circ}$ between the on-source and off-source region. For a source extended on this scale, the off-source region also contains genuine gamma rays, and the background subtraction reduces the signal. A diffuse source generating a uniform distribution cannot be detected by this method. For a halo with an extent of a few degrees, this problem can be circumvented by using background data obtained from a different region of the sky; since these data are usually not recorded under identical conditions, systematic uncertainties in the background estimate will increase.

In the following, we will first examine, with the standard background subtraction, the angular distribution of gamma rays, searching for qualitative indications of a halo. We then use models for the halo angular distribution to quantify the results. Finally, the halo flux is recalculated using a distant background region to cover the case of a halo with a smooth distribution on degree scales.

Any component of the gamma-ray flux which is extended on the scale of a few tenths of a degree or larger should not show any observable time variation, due to the large variation in path lengths. Burst-like emission behavior of the source is completely averaged out. Therefore, the presence of an extended halo component should cause a flux dependence (and time dependence) of the angular distribution of gamma rays; for high flux, the point like source component dominates, whereas for the quiescent flux the extended halo is enhanced.

In a first search for indications of a halo, the ratio of the event rates in different annular regions around the source was studied as a function of time. Specifically, the flux in various angular ranges $\theta_{1}$ to $\theta_{2}$ relative to direction 

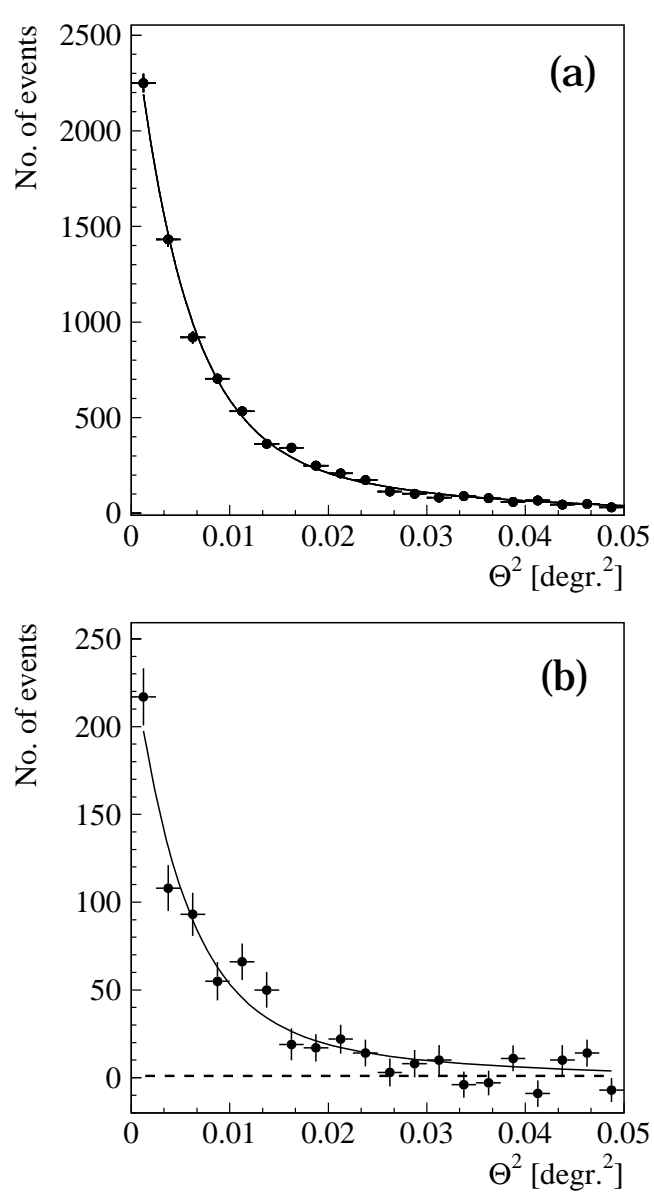

Fig. 1. Angular distribution $\mathrm{d} N / \mathrm{d} \theta^{2}$ of gamma rays relative to the direction of Mkn 501. a) For the 1997 burst period, and b) for the 1998/1999 quiescent state, excluding a short flare in June 1998. Background is subtracted using an off-source region separated by $1^{\circ}$ in declination from the source region. The lines indicate a fit by a superposition of two Gaussian distributions in $\theta$; the same shape is used for both data sets

to Mkn 501 was compared to the flux in the central peak, $\theta<0.05^{\circ} \ldots 0.1^{\circ}$. No statistically significant evidence of a time dependence of the ratios was observed.

In the following, we will concentrate on the flux dependence of angular distributions and specifically compare the high-flux burst periods with the quiescent state of the source. Figure 1 shows the angular distributions of gamma-rays from Mkn 501, after background subtraction, both for the 1997 high-flux burst period (Aharonian et al. 1999a, 1999b), and for the 1998/99 quiescent state (Aharonian et al. 2000a), excluding the flare on June 26-28, 1998 (Sambruna et al. 2000). We note that with the event selection used here, the detection efficiency of the telescope system, i.e. its effective area, is constant to better than $\pm 3 \%$ for $\theta$ between $0^{\circ}$ and $0.5^{\circ}$. In the angular distributions for the high-state and quiescent-state angular distributions (Figs. 1a and 1b, resp.), no dramatic difference is observed. The distributions shown in Fig. 1 are not exactly Gaussian, reflecting the fact that the contributing events have angular resolutions varying between
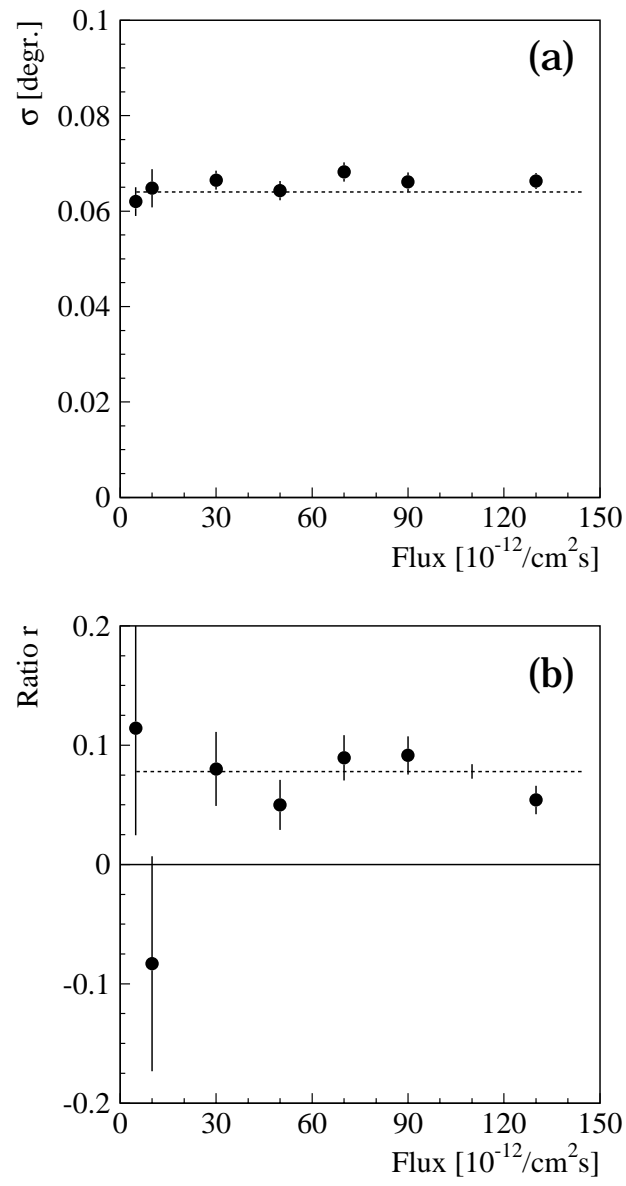

Fig. 2. a) Gaussian width of the angular distribution of gamma-rays relative to the direction to Mkn 501, as a function of the gamma-ray flux. The point for the lowest flux is obtained from the 1998/99 quiescent-state data; the points at higher flux are based on data from the 1997 outburst. The dashed lines indicates the result of the simulation. b) Flux in the angular range $0.22^{\circ}$ to $0.5^{\circ}$ relative to the source, normalized to the flux inside $0.22^{\circ}$. Background is subtracted using an off-source region separated by $1^{\circ}$ in declination from the source region. The error bar on the dashed line indicates the statistical error of the simulation results

$0.04^{\circ}$ and $0.10^{\circ}$. The distributions are well described by a sum of two Gaussian distributions in $\theta$ (solid lines).

To characterize the angular distribution as a function of flux, data were binned according to the average flux measured in each night of observations, and the distribution in $\theta^{2}$ was determined for each bin independently. Six flux bins were used for the 1997 burst data; a seventh "bin" contains the 1998 quiescent-phase data, excluding the June 26-8 flare. The average flux in 1998 is below the flux of the lowest 1997 flux bin. Two quantities characterizing the distribution were examined for each flux bin: the width of the central peak of the distribution, approximated by a single Gaussian (Fig. 2a) and the yield of gamma-ray events detected in the angular range between $0.22^{\circ}$ and $0.50^{\circ}$ from the direction to the source, normalized to the yield inside $0.22^{\circ}$ (Fig. $2 \mathrm{~b}$ ). The first quantity is sensitive to a halo with an angular width on the same scale 


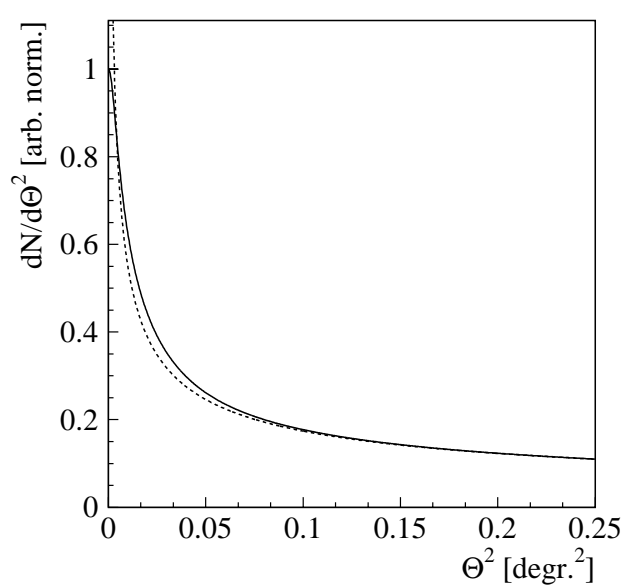

Fig. 3. Expected angular distribution of halo photons for the assumption $N(\theta) \sim \theta$, equivalent to $\mathrm{d} N / \mathrm{d} \theta^{2} \sim 1 / \theta$. The dashed line shows the original distribution, the full line the distribution resulting once the angular resolution of the instrument is folded in

as the angular resolution of the instrument, the second to a halo component with a width significantly larger than the angular resolution. Neither quantity shows a significant dependence on the flux, and the measured values are consistent with results of simulations of the point spread function of the HEGRA instrument, using the measured energy spectrum as an input.

In order to provide a flux limit on the halo flux, a model for the shape of the halo is required. Two variants were investigated.

- A Gaussian distribution, $\mathrm{d} N / \mathrm{d} \theta^{2} \sim \exp \left(-\theta^{2} / 2 \sigma_{\text {Halo }}^{2}\right)$;

- The distribution $\mathrm{d} N / \mathrm{d} \theta^{2} \sim 1 / \theta$ (Fig. 3). The halo simulations of Aharonian et al. (1994, 1995) typically show, for small angles, a distribution $N(\theta) \sim \theta$ where $N(\theta)$ is the number of halo photons inside a cone of opening angle $\theta$, equivalent to $\mathrm{d} N / \mathrm{d} \theta^{2} \sim 1 / \theta$.

To derive limits, the angular distribution of the quiescentstate data was fit by a linear combination of the high-state angular distribution, and a halo contribution. The fit covers the range in $\theta^{2}$ from 0 to $0.25 \mathrm{deg}^{2}$. For none of the halo models, a significant halo contribution was found, and we give in Fig. 4 the $99 \%$ upper limits on the halo flux within an (half-) opening angle of $0.5^{\circ}$ around the source (solid line). The fit and the quoted limits take into account the spill-over of signal photons into the background region for wide halos. The flux limits for the Gaussian halo are lowest for the region of intermediate halo scales, $\sigma_{\text {Halo }} \approx 0.1^{\circ}$, and deteriorate both for narrow halos, which cannot be distinguished from the direct flux, and for very wide halos, where the spill-over into the background region limits the sensitivity. The dashed line in Fig. 4 indicates the limit obtained for the halo model with $N(\theta) \sim \theta$, see Fig. 3. A halo flux corresponding to the limit would roughly triple the expected number of events in the angular range from $0.22^{\circ}$ to $0.5^{\circ}$, see Fig. 2 b. All limits assume that the energy spectrum of cascade photons resembles the spectrum

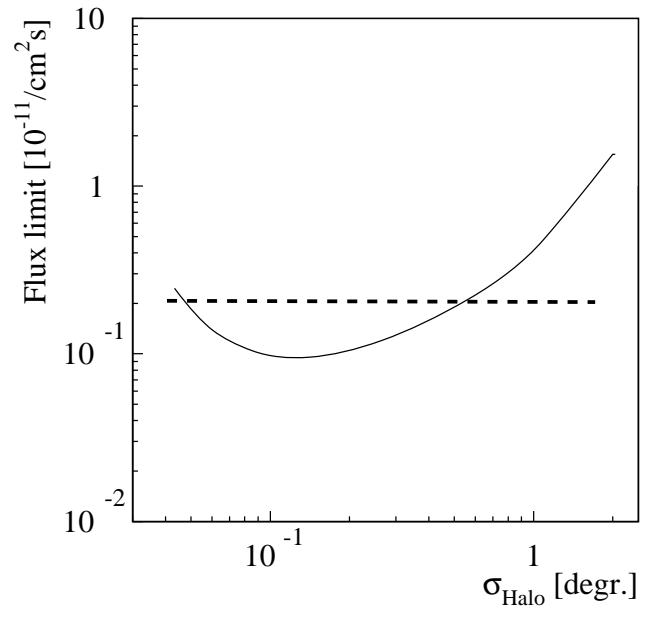

Fig. 4. Full line: upper limit (99\% C.L.) on the halo flux above $1 \mathrm{TeV}$, integrated up to $0.5^{\circ}$ from the source, assuming a Gaussian distribution of halo photons, $\mathrm{d} N / \mathrm{d} \theta^{2} \sim$ $\exp \left(-\theta^{2} / 2 \sigma_{\text {Halo }}^{2}\right)$ as a function of the width $\sigma_{\text {Halo }}$. Dashed line: limit for a distribution $\mathrm{d} N / \mathrm{d} \theta^{2} \sim 1 / \theta$

measured for the quiescent state, i.e. show a power law with an index around 2 and a cutoff at a few $\mathrm{TeV}$.

As discussed earlier, a more model-independent limit on the halo flux can be obtained by using a distant region for background subtraction, rather than the region displaced by only $1^{\circ}$ in declination. Data taken on other objects can, e.g., serve as a background region. The difficulty is that already minor changes in the performance or calibration of the instrument can change the background yield. As an example, Fig. 5a shows the distribution in the mean scaled width of images for a halo region in the Mkn 501 quiescent-state data, and in an equivalent region in Crab data taken at a different time. In this case, the "halo region" was defined as a ring around the source, with an inner radius of $0.33^{\circ}$ and an outer radius of $1^{\circ}$. Together with the required angular resolution of better than $0.1^{\circ}$, the inner cut ensures that virtually no direct gamma-rays spill over into the background region. Figure 5b illustrates, for comparison, a weak gamma-ray signal peaking at a mean scaled width of 1 . While the two distributions shown in Fig. 5a are quite similar, there are differences in the shape, both in the gamma-ray region (mean scaled width between 0.8 and 1.2) and outside. The differences can be explained by systematic shifts of the distribution by as little as 0.01 to 0.02 units, caused e.g. by changes in the alignment of the telescope mirrors with time. The maximum allowable number of gamma-rays in the halo region depends on exactly how the two distributions are normalized to each other, before performing the background subtraction. Worst-case assumptions allow a photon flux of $1.110^{-11} / \mathrm{cm}^{2} \mathrm{~s}$ above $1 \mathrm{TeV}$ in the angular range between $0.33^{\circ}$ and $1.0^{\circ}$ from Mkn 501, equivalent to $1.310^{-8} / \mathrm{cm}^{2} \mathrm{~s}$ sr. Work is in progress to better understand the differences in the distributions, to allow a more reliable background subtraction. 

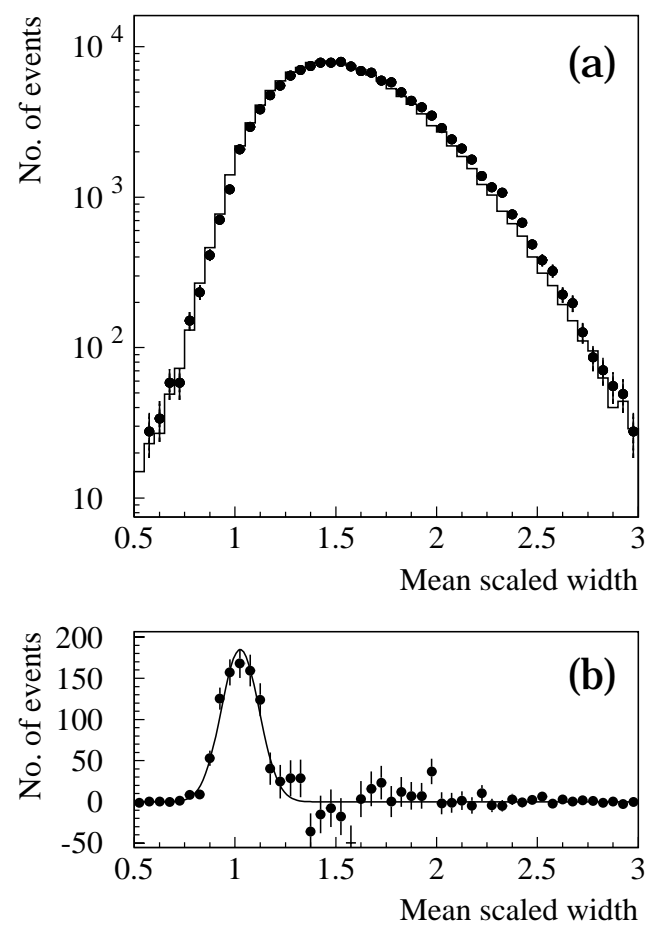

Fig. 5. a) Distribution in the mean scaled width of images in the halo region of the quiescent-state Mkn 501 data sample (full line), compared to the same distribution for the Crab data sample, scaled up to the Mkn 501 data. The halo region covers angles between $0.33^{\circ}$ and $1^{\circ}$ relative to the source. $\mathbf{b}$ ) Distribution for gamma rays, in this case for the quiescent-state Mkn 501 data, after background subtraction

\section{Concluding remarks}

The search for a pair halo surrounding the direct $\mathrm{TeV}$ gamma rays from Mkn 501 shows no indications for a halo; the angular distribution in the quiescent phase - where a halo should be best visible - is within errors identical to the distribution during the burst phases. The upper limits on a halo contribution depend on the model for the angular distribution of halo gamma rays; both very narrow and very wide halos are difficult to detect. In the region of optimal sensitivity, the upper limit on the halo flux within $0.5^{\circ}$ from the source is around $1 / 1000$ of the peak flux during bursts. For conventional halo models (Aharonian et al. 1995, 1994), and "nearby" sources such as Mkn 501, the flux within $0.5^{\circ}$ is $O(10 \%)$ of the total halo flux (for the more realistic "high IR" variant of the model), so that the total halo flux is limited to $O(0.01)$ of the peak burst flux. This value is above the expectations on the basis of energetics, and does not seriously constrain models unless a very large duty cycle or a very poor beaming of the direct radiation is assumed. The situation changes if one assumes the dramatic absorption of gamma rays above $10 \mathrm{TeV}$, as implied by recent measurements of the density of the infrared background radiation; such scenarios imply a huge source power at very high energies and correspondingly a strong halo.
A narrow halo with a width in the $0.1^{\circ}$ range would be generated if intergalactic magnetic fields are very small, in the range below $10^{-16} \mathrm{G}$ (see also Plaga 1995). Detailed cascade simulations will be required to determine which range of model parameters can be excluded; this goes beyond the scope of the present paper.

Acknowledgements. The support of the HEGRA experiment by the German Ministry for Research and Technology BMBF and by the Spanish Research Council CYCIT is acknowledged. We are grateful to the Instituto de Astrofísica de Canarias for the use of the site and for providing excellent working conditions. We gratefully acknowledge the technical support staff of Heidelberg, Kiel, Munich, and Yerevan. GPR acknowledges receipt of a Humboldt Foundation postdoctoral fellowship.

\section{References}

Aharonian, F. A., \& Atoyan, A. M. 1985, Sov. Phys. JETP, 62,189

Aharonian, F. A., Coppi, P. S., \& Völk, H. J. 1994, ApJ, 423, L5

Aharonian, F. A., Coppi, P., Konopelko, A., \& Völk, H. 1995, Proc. of the 24th ICRC, Rome, 2, 511

Aharonian, F. A., Akhperjanian, A. G., Barrio, J. A., et al. 1999a, A\&A, 342, 69

Aharonian, F. A., Akhperjanian, A. G., Barrio, J. A., et al. 1999b, A\&A, 349, 11

Aharonian, F. A., Akhperjanian, A. G., Andronache, M., et al. 1999c, A\&A, 350, 757

Aharonian, F. A., Akhperjanian, A. G., Barrio, J. A., et al. 1999d, ApJ, 359, 317

Aharonian, F. A., Akhperjanian, A. G., Barrio, J. A., et al. 2000a, ApJ, in press [astro-ph/0008211]

Aharonian, F. A., Akhperjanian, A. G., Barrio, J. A., et al. 2000b, A\&A, 361, 1073

Blandford, R. D., \& Levinson, A. 1995, ApJ, 441, 79

Eilek, J. 1999, in Proc. of the Workshop on diffuse thermal and relativistic plasma in galaxy clusters, Ringberg, 71, ed. H. Böhringer, L. Feretti, \& P. Schuecker

Goldreich, P., \& Morrison, P. 1964, Sov. Phys. JTEP, 18, 239

Gould, J., \& Schreder, G. 1966, PRL, 16, 252

Gould, J., \& Schreder, G. 1967, PR, 155, 1404

Hofmann, W., Jung, I., Konopelko, A., et al. 2000, Astropart. Phys., 12, 135

Jelley, J. V. 1966, PRL, 16, 479

Konopelko, A., Hemberger, M., Aharonian, F., et al. 1999, Astropart. Phys., 10, 275

Kronberg, P. P. 1995, Nature, 374, 404

Kronberg, P. P. 1996, Space Sci. Rev., 75, 387

Nikishov, A. I. 1962, Sov. Phys. JETP, 14, 393

Plaga, R. 1995, Nat, 374, 430

Protheroe, R. J. 1986, MNRAS, 221, 769

Protheroe, R. J., \& Stanev, T. 1993, MNRAS, 264, 191

Protheroe, R. J., \& Meyer, H. 2000 [astro-ph/0005349]

Sambruna, Aharonian, F. A., Krawczynski, H., et al. 2000, ApJ, 538, 127

Stecker, F. W., De Jager, O. C., \& Salomon, M. H. 1992, ApJ, 390, L49

Zdziarski, A. J. 1988, ApJ, 335, 786 